\title{
An Empirical Study of Geography Learning on Students' Emotions and Motivation in Immersive Virtual Reality
}

\author{
Yang Shen', Zhaoxue Wang' ${ }^{2}$, Ming $\mathrm{Li}^{3}$, Jing Yuan ${ }^{4}$ and Yunchao Gu${ }^{3 *}$ \\ ${ }^{1}$ Collaborative Innovation Center of Assessment Toward Basic Education Quality, Beijing Normal University, Beijing, China, \\ ${ }^{2}$ Faculty of Education, Beijing Normal University, Beijing, China, ${ }^{3}$ State Key Laboratory of Virtual Reality Technology \\ and Systems, Beihang University, Beijing, China, ${ }^{4}$ Quality-Oriented Education Research Center, Anhui Xinhua University, \\ Hefei, China
}

OPEN ACCESS

Edited by: Xuesong Zhai,

Zhejiang University, China

Reviewed by:

Zhanpeng Jin

University at Buffalo, United States

Xiaosong Yang, Bournemouth University,

United Kingdom

*Correspondence:

Yunchao Gu

guyunchao@buaa.edu.cn

Specialty section:

This article was submitted to Educational Psychology,

a section of the journal

Frontiers in Education

Received: 08 December 2021

Accepted: 31 January 2022

Published: 23 February 2022

Citation:

Shen Y, Wang Z, Li M, Yuan J and

Gu Y (2022) An Empirical Study of Geography Learning on Students' Emotions and Motivation in Immersive Virtual Reality. Front. Educ. 7:831619.

doi: 10.3389/feduc.2022.831619
This paper assesses the impact of emotional arousal on learning through a virtual reality video of geography immersion learning. Validity was measured with traditional questionnaire data and using electroencephalography (EEG). Twenty-four human subjects were recruited and presented with eight geography immersive learning videos with different affective tendencies. EEG data of the subjects were collected while they were watching the videos. After the video watching, subjects were requested to fill in an emotion scale, a learning motivation scale, and a mind flow experience. The results of the study show that VR video learning materials can well induce the positive and negative emotions of the subjects. Compared with negative emotions, the alpha band power in the frontal lobe of the brain, beta and gamma bands in the temporal lobe region is significantly higher under positive emotions. In addition, the results of the subjective scales indicate that subjects have stronger intrinsic motivations and better flow experiences under positive emotions. However, there was no significant difference for the immersion under positive and negative emotions. Our findings demonstrate the usability of teaching virtual reality situational geography stories and the broad value of using this teaching method for future instruction.

Keywords: virtual reality, immersive learning, EEG, emotion, motivation

\section{INTRODUCTION}

Virtual reality technology has revolutionized traditional education, which involves medicine, architecture, and other disciplines (Shen et al., 2021). With the superior advantages of immersion and interaction, virtual reality can be seamlessly integrated into geography teaching to build an immersive virtual geography learning environment for learners. In traditional geography teaching, due to various factors, students cannot easily go out of campus for geography study activities. Thus, students can't access the field scenes and lack on-site practice, not to mention the in-depth understanding and re-creation of knowledge (Thomas et al., 2018). Virtual Reality (VR) immersion learning, on the other hand, is a concept that combines groundbreaking virtual reality technology and traditional narrative lecturing. The virtual reality-based 3D animation technology simulates and demonstrates the process of movement and change of geographical phenomena by the laws of geography. Students are immersed in the colorful and dynamic surrounding scenes and feel the thousands of changes in the laws of geography. It enhances students' emotional experience, 
which initiates learners' learning motivation and thus enhances learners' learning effect. Emotion elicitation, as an essential instructional design tool in multimedia teaching, can effectively induce learners' emotions and may have an impact on their learning outcomes and subjective feelings (Park et al., 2015; Kumar et al., 2016; Münchow et al., 2017). However, there is controversy among the available research findings on how evoked emotions affect learners' learning performance and subjective feelings. And some studies have shown that immersive learning is very effective in developing students' learning skills and motivation (Moreau et al., 2018). Therefore, based on an immersive geography immersive learning teaching activity to elicit learners' emotions, this study aims to use more realistic and objective physiological data of brain waves to characterize emotions and investigate the emotion-evoking effects of this learning style on learners. It also investigates the relationship between emotion-evoking and factors, such as learning motivation and mind-flow experience, to conduct virtual reality emotion-evoking in future virtual reality immersive learning teaching design.

In the present study, three research questions were presented and addressed.

Hypothesis 1: VR immersive learning in a virtual reality environment can have an evocative effect on learners' emotions.

Hypothesis 2: VR immersive learning in a virtual reality environment can have an impact on learners' motivation levels.

Hypothesis 3: There is a correlation between emotion and motivation, flow experience, and immersion levels, respectively, for immersive learning in a virtual reality environment.

\section{LITERATURE REVIEW}

\section{Immersive Virtual Reality Learning What Is Immersive Virtual Reality Learning}

Immersive virtual reality learning involves user-centered engagement with narratives that unfold as witnesses or participants (Dooley, 2017). Immersive virtual reality learning offers a rare opportunity to present abstract experiences that challenge boundaries, heighten emotions, and convey previously intangible concepts (Hardie et al., 2020). This new approach to immersive virtual reality geography learning differs from traditional narrative media, such as film and television, in which the audience is mostly a passive participant. Immersive virtual reality learning is not about teaching the audience something as it is about allowing the audience to discover the knowledge (Bucher, 2017).

\section{Educational Applications of Geography Learning in Immersive Virtual Reality}

In recent years, immersive learning in virtual reality has also received much attention as a potential new tool for science education (Zhai et al., 2021b). VR immersive environment provides a unique opportunity to present abstract experiences that push boundaries, stimulate learners' emotions, and explain previously incomprehensible concepts (Bucher, 2017). However, most of the current educational applications for immersive learning under virtual exist in healthcare and education, and less for geoscience.

However, immersive geography learning is also particularly important in earth science. On the one hand, real-life experience is an important part of geography teaching. Geography course is a very comprehensive and life course, the course itself covers the geographical knowledge content and the geographical environment of students' actual life is closely related, from a drop of water, a piece of soil, to the famous mountains and rivers of the motherland, are all students need to pay attention to when learning geography courses. In traditional classrooms, it seems impossible for teachers to take students to all geographic environments due to other factors such as funding, time, and safety. Instead, with VR devices, students can experience some geographic processes and geographic environments that they cannot experience in real life (Bucher, 2017). For example, Virvou and Katsionis (2008) use virtual geography games for classroom teaching, teaching students geography, and then making evaluation experiments. This way of teaching virtual reality games allows students to gain knowledge through experience and promotes the development of students. Professor Yukiko Inoue conducted an experimental survey using virtual reality technology and video teaching to evaluate the effectiveness of the classroom when high school students learned about world geography (Inoue, 1999).

On the other hand, interactive experience is an important way in geography teaching, based on virtual reality interactive technology and by the content of geographic experiments, the creation of human-landscape interactive virtual geographic experiments (such as virtual globes, virtual solar system, etc.), so that students through hands-on experiments, enhance the contextual experience and interactive experience of geography learning-this to a certain extent make up for the current geography teaching through multimedia courseware and teaching aids model experiments exist in the situational interaction limitations. In immersive virtual reality learning, learners follow narrative stories presented by the teacher, which integrates storytelling and interactivity into an engaging learning scenario. Combining geographic narratives with virtual reality can create memorable experiences that evoke emotions and make it easier for learners to remember due to the power of its sensory associations. Scientific research in immersive VR learning is still in its infancy, especially in the area of geography and natural science narratives (Li et al., 2007).

\section{Emotional Arousal}

Emotion is a coping response used to help individuals better adapt to the environment they are in Roseman (1996), and research has proven that the emotions of disgust, fear, happiness, surprise, sadness, and anger are common in life (Ekman, 1992). Mayer (2010) points out that there are still certain moderating variables or boundary conditions in the practical application 
of multimedia teaching principles, namely multimedia learning principles may only be more effective if they are targeted to specific learners and if certain specific design approaches or learning materials are used (Zhai et al., 2021a).

The external emotion elicitation methods used in previous empirical studies usually contain video and non-video (e.g., music, declarative sentences, etc.) As the two types of elicitation (Knörzer et al., 2016; Liew and Tan, 2016), but compared to this, immersive virtual reality can make full use of the psychological and physiological components of the subject, thus inducing learners' emotions more completely (Pallavicini and Pepe, 2020). Thus, with its three-dimensional and realistic environment, virtual reality allows users to enjoy an immersive and intuitive sensation and is the superior emotion-evoking meta.

\section{The Relationship Between Emotional Triggers and Motivation}

In the study of emotion-inducing effects, researchers not only paid attention to the effect of induced emotions on learning effects but also paid attention to the changes in the subjective feelings of learners in multimedia learning. Based on previous studies, studies on emotion induction have focused on intrinsic motivation as an indicator (Shen et al., 2021).

Eisenberg et al. (1992) and Vansteenkiste et al. (2012) argue that motivation is a continuum of intrinsic motivation to extrinsic motivation. Based on this view, Weiner (1986) and Chandler and Connell (1987) divide motivation into intrinsic motivation, internalized motivation, and extrinsic motivation. Among them, internalized motivation refers to the intrinsic identification and pursuit of the meaning of learning activities by external factors, and becomes the leading driving force for learning. Motivation is a significant cognitive factor that influences learning; therefore, better-motivated students can learn more effectively (Cobb and Fraser, 2005). Researchers have studied the relationship between emotion and motivation during learning, but the results vary. Mayer (2014) states that performing emotional design is an attempt to combine motivation with cognition. That is, emotional design motivates learners to put more mental effort, which will result in higher motivation for positive processing and ultimately lead to better learning outcomes. In terms of intrinsic motivation, Mayer (2013) used a combination of external emotion elicitation and internal emotion elicitation and found that the positive emotion elicitation group was more intrinsically motivated than the neutral group. Plass et al. (2014) also used external emotion elicitation and internal emotion elicitation but only found that the internal positive emotion elicitation group was more intrinsically motivated than the internal neutral emotion elicitation group. In contrast, Kumar et al. (2016), who also used only internal emotion elicitation methods, found that emotion elicitation did not affect learners' internal motivation. Pavelescu (2019) explored the relationship between motivation and affective factors through two contrasting cases, demonstrating that strong positive emotions can enhance and sustain motivation. Although emotion elicitation and motivation in learning experiences have been extensively studied, the relationship between motivation and emotion in virtual reality has not been thoroughly researched.
Limniou et al. (2008) showed that 3D fully immersive VRLE would increase learners' interest and motivation compared to learning in a 2D animated environment. Burdea and Coiffet (2003) suggested that through interaction and potentially high repetition, virtual reality helps to increase knowledge retention and positive student emotions and motivation.

\section{Measurement}

\section{Measurement of Motivation}

Objective or quantitative assessment of learners' motivations represents one of the most critical tasks in this research, to better understand and gauge the effects of the proposed VR-based learning approach. There have been various prior research efforts aiming to provide solutions for this complex task. Different methodologies were proposed (Ghergulescu and Muntean, 2014) to measure and assess a learner's motivation:

(i) Questionnaire-based methodology;

(ii) Interaction-based methodology;

(iii) Psychophysiology-based methodology.

Motivation measurement and assessment based on physiology and psychology rely on additional devices such as behavioral data (facial expressions, eye movements) and data on physiological responses (heart rate, brain signals, and skin electricity). Kleih et al. (2010) investigated the relationship between motivation and P300 (an event-related potential). Pi et al. (2021) proposed electroencephalography (EEG)-based measure of motivation for learning in a video learning environment $\mathrm{g}$. The participants had to perform a spelling words task. Furthermore, the results have shown that motivation can be significantly predicted by P300. Therefore, psychophysiological-based methodologies that make use of sensor-based recognition are preferred as they usually do not require an interruption of the learning process (Ghergulescu and Muntean, 2014).

\section{Measurement of Emotions}

Facial expressions, voice intonation, gestures, and physiological signals can all be used as data sources for emotion evaluation. However, sometimes the emotional state can be disguised. For example, one person is angry inside, but he expresses a happy expression for social reasons. Therefore, in these situations, facial expressions and voice intonations cannot accurately recognize emotions. In addition, physiological signals are difficult to disguise and contain more information.

In this paper, we use physiological signals and subjective scale data to measure emotions. These two types of data are important indicators for evaluating emotions, some studies have shown differences between physiological signals and subjective scales under different emotions (Zheng and Lu, 2015). It is well known that neural oscillations in the theta frequency band $(4-8 \mathrm{~Hz})$ are associated with memory (Herweg et al., 2020).

\section{MATERIALS AND METHODS}

\section{Participants}

24 healthy participants (14 males and 10 females), aged between 21 and 33 were recruited for this study, and all participants 
had a normal or corrected-to-normal vision. None of them had a history of heart disease, neurological disorders, or other mood-related disorders. The local ethics committee has approved the investigation.

\section{Instruments Measuring Tool}

\section{The Self-Assessment Manikins Questionnaire}

In this experiment, we use the Self-Assessment Manikins (SAM). The scale contains information such as Arousal, Valence, Dominance, and Liking. Here we mainly collect the subjects' arousal scores and valence scores, which correspond to the twodimensional model of emotions.

The horizontal and vertical axes of this model represent the degree of valence and arousal, respectively. The valence represents the degree of pleasure of the emotion, arousal represents the intensity of the emotion. The four poles of the model represent four different discrete emotion categories: happiness (high valence/High arousal), ease (high valence/low arousal), boredom (low valence/low arousal), panic (low valence/high arousal). In the literature, this two-dimensional representation model is often called VA (Valence-Arousal) Model

\section{The Learning Motivation Scale}

The learning motivation measure was developed by Wang and Chen (2010) based on the measure proposed by Pintrich (1991). Six items were included to measure learners' internal and external motivations in virtual reality immersion learning.

\section{Virtual Reality Immersive Immersion Scale}

Huang et al. (2010) developed this instrument to investigate learners' attitudes toward virtual reality learning environments (VRLE), which is based on a constructivist theoretical approach that examines students' interaction, immersion, imagination, motivation, and problem-solving with virtual reality learning environments.

\section{Flow Experience Scale}

Flow is defined as a psychological experience reflecting cognitive regulation in behaviors (Chen, 2007; Csikszentmihalyi, 2014). Flow theory focuses on the emotional experiences of individuals, focusing on the generation of internal motivations that drive individuals to engage in an activity. Once students have a flow experience in the geography classroom, they will devote themselves to geography learning with great enthusiasm, transforming external motivations into internal motivations, and ultimately forming a virtuous circle. This study, based on Cheng's scale of the virtual reality game, adopted a five-point scale of the virtual reality environment, including four dimensions: namely challenge, skill, telepresence, flow. They represent the challenges faced in the learning process, the skills learned in this teaching method, and the selfless realm in learning and a state of mind sometimes experienced by people who are deeply involved in this activity. After modifying the scale according to the immersive learning environment of virtual reality, we used the modified version for pre-testing, reliability testing, and validity testing. We conducted project analysis, reliability, and validity analysis of the data. The scale has good reliability and can be used in VR geography Learning.

\section{Emotional Evocation Video Selection}

This research uses immersive virtual reality videos as the primary teaching media learning environment and immersive geography videos as the main content. The video comes from the Stanford immersive VR video public database ( $\mathrm{Li}$ et al., 2017). The dataset contains 73 immersive VR videos, and each video has a corresponding valence and arousal score, which are distributed in the four quadrants of the arousal valence plane ( $\mathrm{V}$ plane). We selected virtual reality videos consistent with the content of compulsory high school textbooks and described the virtual scenes. Sample screenshots of VR videos are shown in Figure 1. We then merged the described voice description with the virtual video to form a supporting virtual reality immersive geography video. The average duration of each video is $300 \mathrm{~s}$. The emotional categories and topics of VR videos are shown in Table 1.

\section{The Measurements of Brain Waves}

Our experimental equipment consists of the following parts: a computer (Intel Core i7 processor, GTX 2060 graphics card, and 16GB RAM), an HTC Vive Pro HMD for displaying VR videos. We measured and set the interpupillary distance for each participant. EEG signals were collected by the ANT Neuro equipment, whose electrode placement follows the 10/20 system. Besides, to prevent the head-mounted device (HMD) from applying pressure on the frontocentral electrodes, a lateral elastic band is used to fix the HMD while the upper elastic band is loose. The experiment recorded a total of thirty-two (32) EEG channels, which is the same as the DEAP and MAHNOB-HCI datasets. As shown in Figure 2, similar to the DEAP and MAHNOB-HCI datasets, all electrodes were grounded to the forehead, referenced to $\mathrm{CPz}$, and then re-referenced to the linked papillae offline. The HMD and EEG caps used in this experiment are shown in Figure 3.

\section{Statistical Analysis Method}

In this experiment, we used the non-parametric Wilcoxon signed-rank test to analyze the differences in learning motivation and flow experience under different emotional states. This analysis method does not require the data to follow a normal distribution. The significance level in all experiments is 0.05 .

\section{Procedure}

The experimental, temperature-controlled laboratory was set at a constant temperature of $20^{\circ} \mathrm{C}$. The windowless lab also allowed the complete darkness in the room to minimize distractions and avoid light affecting the quality of the projected image. As shown in Figure 4, the study consisted of the following three steps. In the first step, all participants washed their hair to lower the impedance. Then, they filled out the demographic information form (e.g., age, gender). In Step Two, the experimental subjects wore HMD and watched eight virtual reality geographic videos in a random order. At the end of the video viewing, participants completed psychometric questionnaires on motivation to learn, 

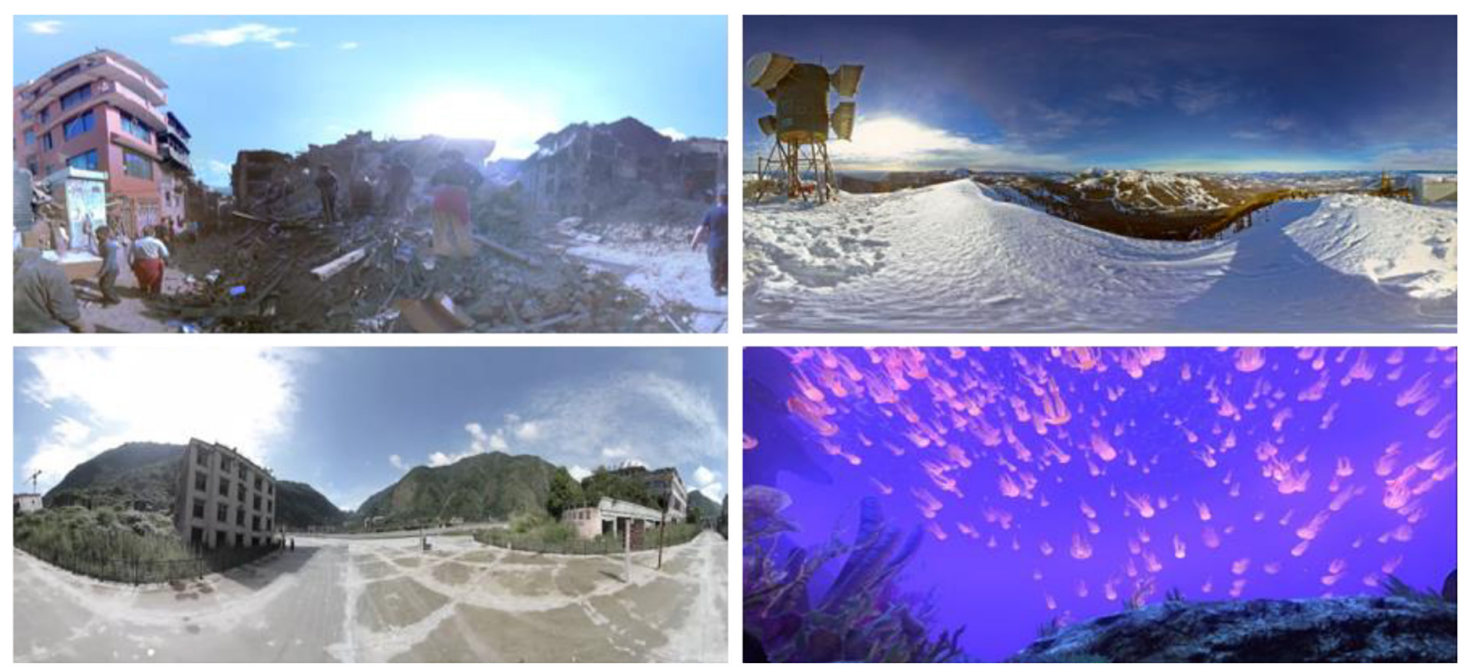

FIGURE 1 | Sample screenshots of VR videos with different emotions they watched.

TABLE 1 | Video topics.

\begin{tabular}{|c|c|c|c|}
\hline Emotional & & Video topics & Video content \\
\hline \multirow[t]{4}{*}{ Positive emotions } & Delighted & Human geography & Foreign urban structure \\
\hline & & & $\begin{array}{l}\text { Appreciation of our human } \\
\text { tourism resources }\end{array}$ \\
\hline & Excitement & $\begin{array}{l}\text { Environmental } \\
\text { protection }\end{array}$ & Whale Encounters \\
\hline & & & Coral reef migrations \\
\hline \multirow[t]{4}{*}{ Negative emotion } & Phobia & $\begin{array}{l}\text { Natural disasters } \\
\text { and their impact }\end{array}$ & Nepal earthquake \\
\hline & & & Turmoil \\
\hline & Sorrowful & $\begin{array}{l}\text { Earth in the } \\
\text { Universe }\end{array}$ & Volcanic eruption \\
\hline & & & Flooding outbreak \\
\hline
\end{tabular}

mind-flow experience, and emotions. The entire experiment lasted approximately $2 \mathrm{~h}$.

\section{Data Analysis}

In this section, we analyze the results from five aspects, including the effect of positive and negative emotions on students' motivation, flow experience, and immersion was investigated through subjective scale scores and objective physiological data.

\section{Analysis of Self-Assessment Manikins Questionnaire Ratings}

We calculate the average SAM ratings of eight VR videos watched by 24 subjects. The results are shown in Table 2. For four negative VR videos, the valence scores are generally lower: 3.1, 3.2, 2.3, and 2.1 , respectively. For the four VR videos with positive emotions, the corresponding valence scores are generally higher: 6.7, 6.8, 6.6 , and 7.9 , respectively.

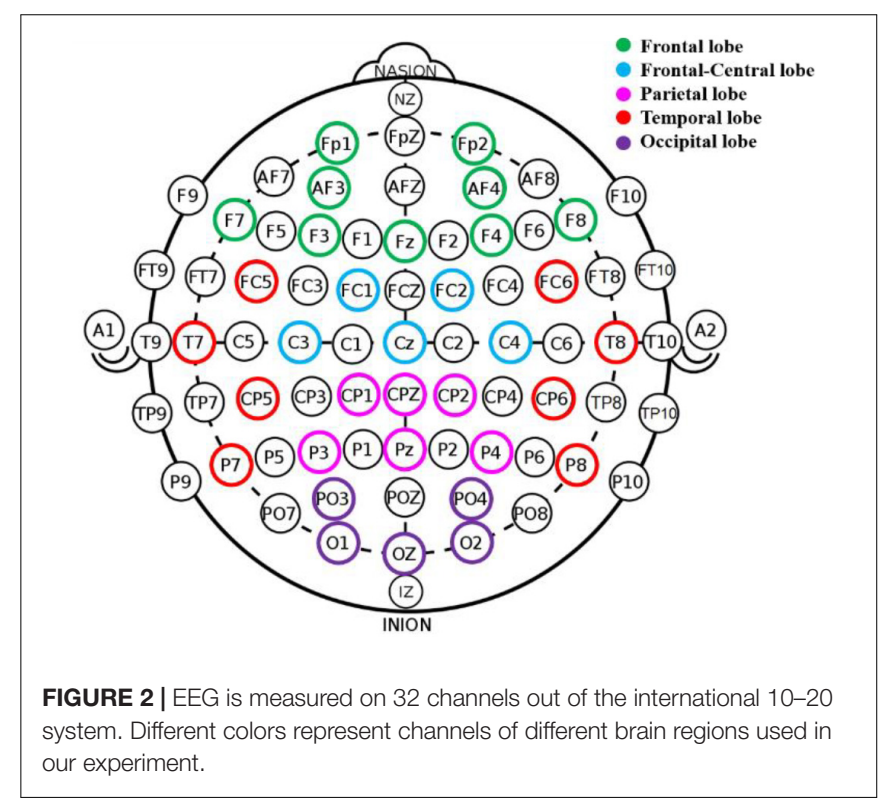

Then, we compare the arousal and valence scores of 24 subjects under positive and negative emotions. The nonparametric Wilcoxon signed-rank test analyzes statistical differences between these two emotional states. As shown from Figure 5, we can see a significant difference $(p<0.001)$ for valence scores between positive (6.99) and negative (2.68) emotions. But there has no significant difference for arousal scores under positive (5.68) and negative (5.38) emotions. The reason for these results may be the subjects were able to immerse themselves in the VR scene and complete the learning task well regardless of whether they were in negative or positive emotions. So there is no significant difference in arousal scores. In short, the results suggest that the eight VR videos, as learning materials in the 


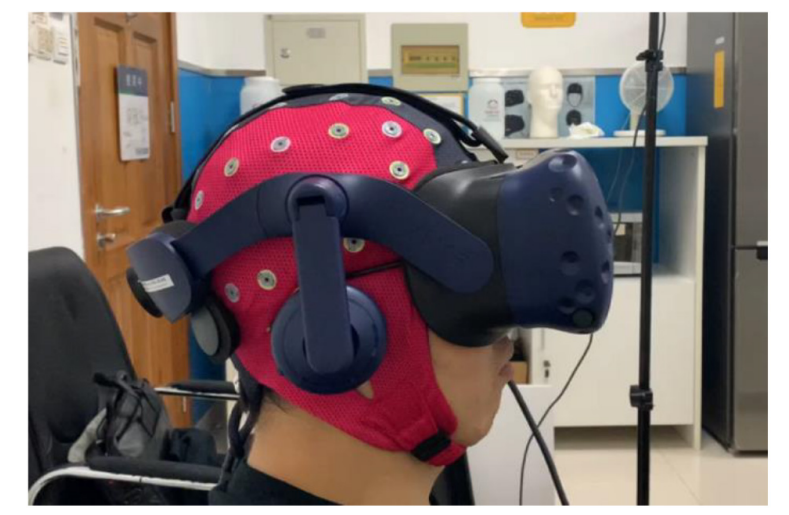

FIGURE 3 | EEG equipment was used in our study.

experiments, successfully induced the positive and negative emotions of the subjects, which supports our Hypothesis 1.

\section{Electroencephalography Frequency Feature Analysis}

To compare the difference of EEG frequency features between positive and negative emotional states. We first resampled the EEG data to $256 \mathrm{~Hz}$. Python's MNE package was used to filter the signal and keep the data only between 0.1 and $47 \mathrm{~Hz}$ frequency band. Then we used the independent component analysis (ICA) to remove electrooculogram (EOG) artifacts. Finally, the Welch method was used to extract the power spectral density (PSD) features over the five bands of the EEG signal delta, theta, alpha, beta, and gamma. These power features were averaged on the corresponding waveband and then were also averaged over all electrode channels.

The statistical analysis results through the non-parametric Wilcoxon signed-rank test are shown in Figure 6. There has a significant difference for the PSD features of alpha-band over the forehead region. The alpha-band feature values under
TABLE 2 | Eight VR videos' mean valence and arousal ratings (standard deviations).

\begin{tabular}{lcc}
\hline Videos & Valence & Arousal \\
\hline Nepal earthquake & $3.1(0.5)$ & $4.5(0.4)$ \\
Turmoil & $3.2(0.8)$ & $4.8(0.3)$ \\
Volcanic eruption & $2.3(0.4)$ & $8.1(1.2)$ \\
Flooding outbreak & $2.1(0.2)$ & $8.2(1.3)$ \\
Foreign urban structure & $6.7(0.9)$ & $4.1(0.5)$ \\
Appreciation of our human tourism resources & $6.8(1.0)$ & $4.0(0.3)$ \\
Whale encounters & $6.6(0.8)$ & $7.4(0.9)$ \\
Coral reef migrations & $7.9(1.0)$ & $7.2(0.7)$
\end{tabular}

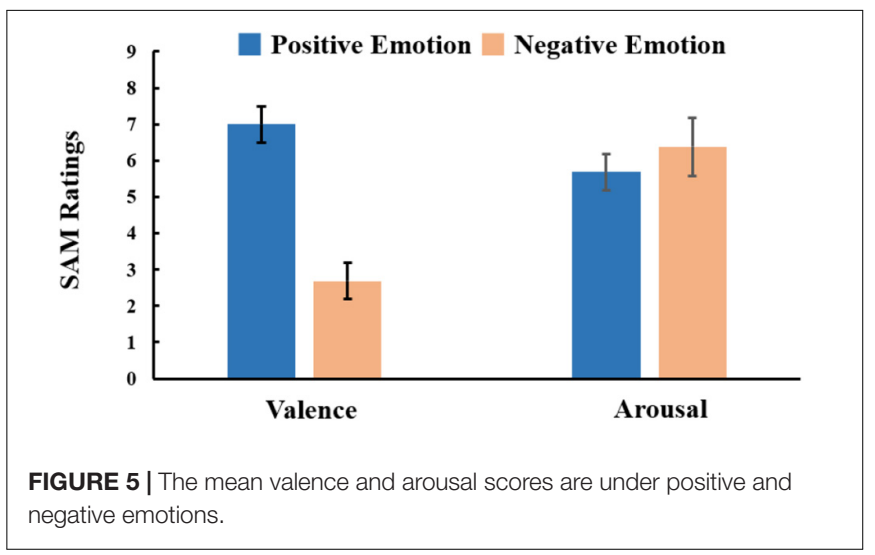

positive emotions are significantly higher than the values under negative emotions $(p<0.01)$. In addition, the PSD features are significantly different for the beta-band of the temporal lobe. The feature values under positive emotions are significantly higher than those under negative emotions $(p<0.001)$. Similarly, as shown in Figure 6, for the gamma-band of the temporal lobe, the values of the features under positive emotions are also significantly higher than the values of negative emotions.

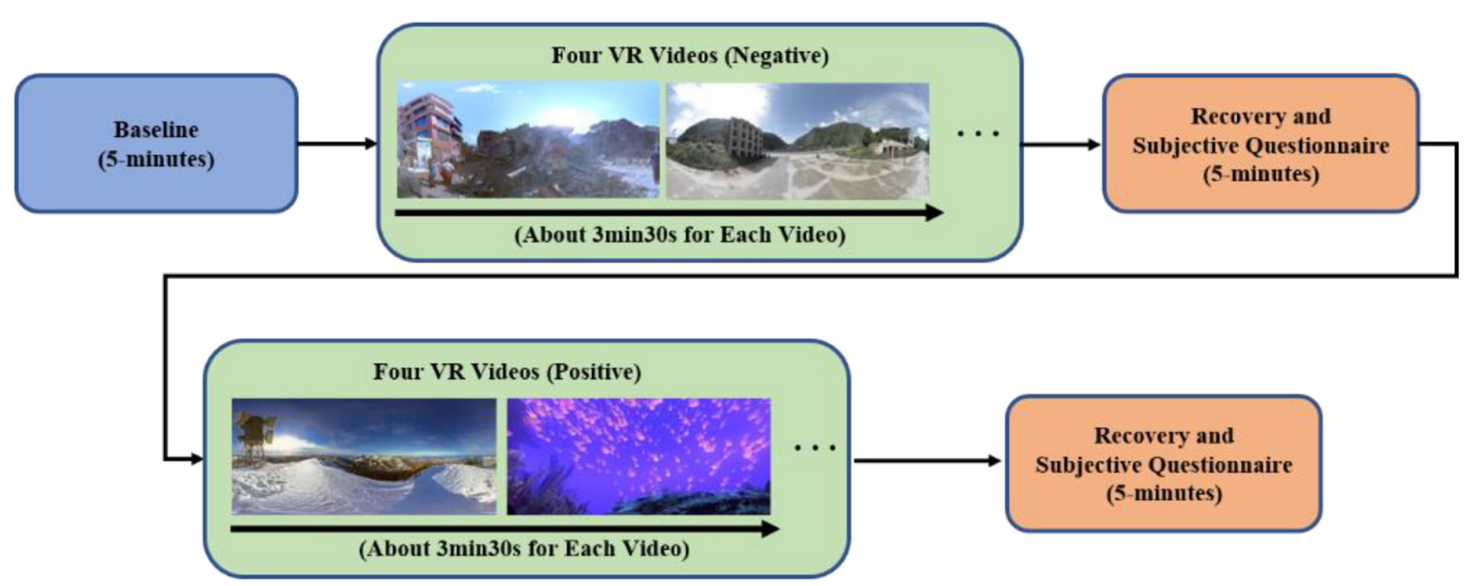

FIGURE 4 | The experimental design of our study. 

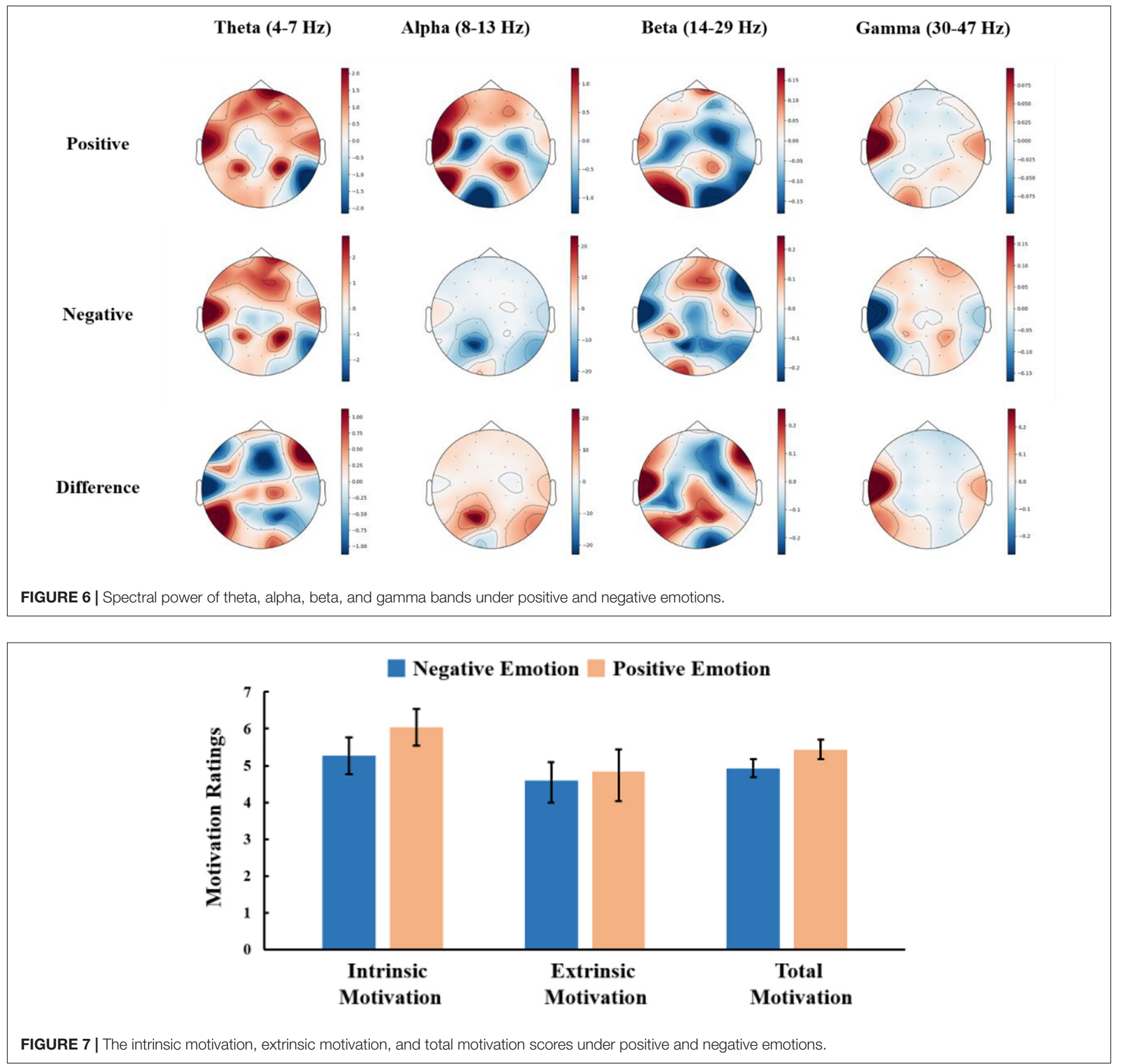

\section{Analysis of Learning Motivation Under Positive and Negative Emotions}

To study the level of extrinsic motivation, intrinsic motivation, and total motivation under different emotions, we use the nonparametric Wilcoxon signed-rank test that was conducted on each of the six items on the learning motivation scale.

The descriptive results are shown in Figure 7. Only intrinsic motivation has a significant difference between positive and negative emotions $(p<0.01)$. Subjects have greater determination and motivation to complete learning tasks under positive emotions. Regarding extrinsic motivation and total motivation, positive emotions and negative emotions have no significant difference. We believe the reason for this phenomenon is that most of the subjects use VR as learning material for the first time, and they are generally excited. Therefore, in future work, we will let the subjects familiarize themselves with VR before the experiment begins. This kind of "warm-up" session may help eliminate the "first excitement." Even if they have negative emotions, they can still maintain sufficient motivation to participate in the learning task. This result supports Hypothesis 2. It is also evident from the study that the novelty of virtual reality technology as a multi-sensor user interface promotes learners' motivations by representing personality traits, engaging media, and stimulating dialogue (Huang et al., 2010). 
TABLE 3 | The mean flow experience, immersion ratings, and standard deviations under negative and positive emotions.

\begin{tabular}{lcccc}
\hline Score & Condition & $\boldsymbol{n}$ & Mean & SD \\
\hline Flow experience & Negative emotion & 24 & 3.750 & 0.386 \\
& Positive emotion & 24 & 4.196 & 0.793 \\
Immersion & Negative emotion & 24 & 3.928 & 0.788 \\
& Positive emotion & 24 & 4.250 & 0.537 \\
\hline
\end{tabular}

\section{Analysis of Flow Experience Under Positive and Negative Emotion}

We also examined the influence of 24 subjects' flow experience under positive and negative emotions. The results are shown in Table 3, the average total flow experience score is 4.196 $(S D=0.386)$ for positive emotions, and $3.750(S D=0.793)$ for negative emotions. The results of the non-parametric Wilcoxon signed-rank test show a significant difference in the flow experience under these two emotional states $(p<0.05)$.

\section{Analysis of Immersion Under Positive and Negative Emotions}

The influence of 24 subjects' immersion under positive and negative emotions is also shown in Table 3. It can be seen that the average total immersion score is $4.250(S D=0.537)$ for positive emotions and 3.928 ( $S D=0.788)$ for negative emotions. Also using the non-parametric Wilcoxon signed-rank test, the results suggest that there is no significant difference in the immersion under these two emotional states. The reason for this result is that the subjects watched the video learning materials from the first perspective, and the immersion in VR is generally high. Therefore, positive emotions and negative emotions have little effect on the subjects' immersion experience.

\section{Relation Analysis of Emotion and Motivation, Flow Experience, and Immersion}

To study whether positive and negative emotions are related to motivation, flow experience, and immersion. We analyzed the Spearman correlation coefficients between the valence scores and total motivation, flow experience, and immersion scores of 8 videos. Valence scores are used as the independent variables, and three experience scores are used as the dependent variables. As shown in Figure 8, there is a certain correlation between the valence and the three experience scores. With the increase in video valence scores, the three experience scores also show an increasing trend. Among them, the Spearman correlation coefficients between the valence and a specific experience score are $r=0.530$ for the total motivation, $r=0.542$ for the flow experience, and $r=0.503$ for the immersion, respectively. This result supports Hypothesis 3.

\section{Analysis of Interviews}

The researcher used both unstructured and semi-structured interviews with college students majoring in geography. In general, learners had good feedback on the VR immersion learning approach.

In terms of learning content, learners generally found that they could learn and understand the knowledge in a more specialized lesson using the VR immersion learning method of teaching. Students had a strong visual experience of learning this component through VR immersion learning. When they recalled the course content, they said that they could make a connection between the text content of the lecture and the virtual reality video, so it created a deep memory, which was helpful for them to review the knowledge of the lesson and learn new knowledge. This has similar findings to a previous study by Rauch (2007) who concluded that "realistic, interactive digital characters, as instructors and role-players, have been shown to significantly increase learner motivation and retention." This study supports these findings, as students expressed that VR immersion learning experience improved their comprehension, sequencing, and recall of abstract concepts (Alfalah et al., 2019). Essentially, both personal and emotional stories engage the brain and are therefore easier to remember than just stating facts. They expressed strong interest in using this approach to learn again if given the opportunity.

In terms of learning methods, in the previous geography learning, students acquired indirect experience based on textbooks and teachers' words, and they found it difficult to understand the acquisition of direct experience which was often difficult to achieve due to the teaching conditions and class time constraints. Students indicated that the VR immersion learning approach allows direct and indirect experiences to be blended, with much less restrictive factors than the traditional learning
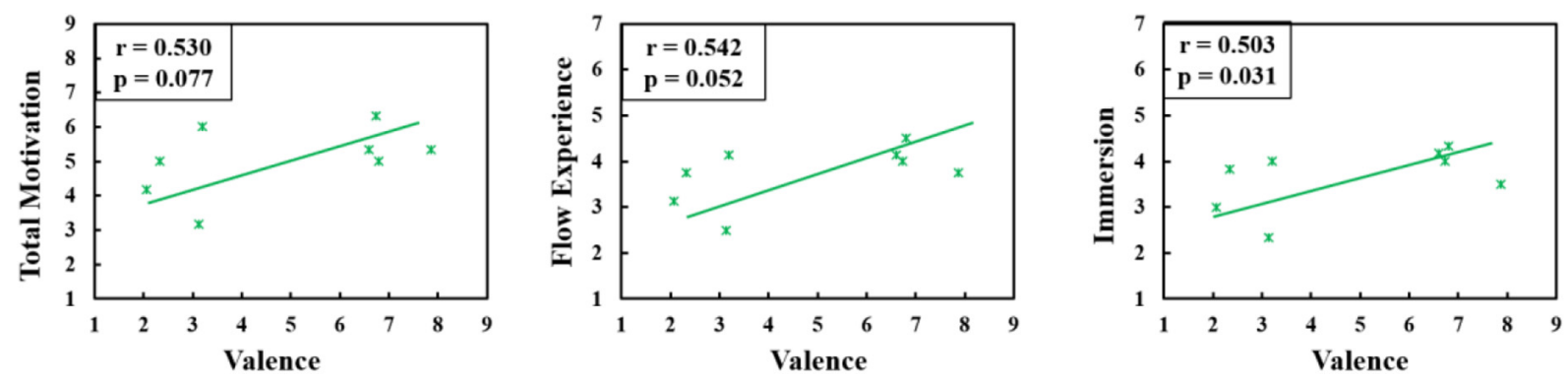

FIGURE $\mathbf{8}$ | The Immersion scores under positive and negative emotions. 
methods to which they are normally subjected. Learners can access diverse information from multiple sources in such an environment, thus maintaining a sense of enjoyment, focus, control, and immersion in learning.

Furthermore, a small proportion of students also presented a negative review of the VR immersion learning experience. Specifically, as Suh and Prophet (2018), a few students reported similar findings citing immersive technology did not always create a positive learning experience and this study caused motion sickness to them. It is thus necessary to determine what features of the VR immersion learning experience influenced the participants' viewing behavior and the degree of emotional arousal and specific triggers for motion sickness.

\section{DISCUSSION}

\section{Presents an Immersive Virtual Reality Geography Landscape That Mobilizes a Positive Experience in the Geography Classroom}

Firstly, virtual reality immersive geography teaching could eliminate the distance between students and the geography environment. The distant geographic landscape becomes accessible to meet the curiosity and fascination of students with different geographic scenery. Secondly, through virtual reality technology, students immerse themselves in the visual, auditory, and other senses into the realities of different landscapes and fully experience the gorgeous, dreamy, and strange geographical landscapes. At the same time, these sensory triggers can help the listeners/viewers of the stories understand the nature of complex concepts and ideas in a more accessible and effective manner (Storr, 2020). Through data analysis, it is found that immersive geography learning is a teaching tool that elicits an emotional response and captures students' attention, thus stimulating their interest in learning. In addition, we found that positive emotions elicited more interest, motivation, and immersion in learning than negative emotions during the learning process.

\section{The New Method Measures Emotional Perception During Learning}

Although the emotional feelings of learners can be measured to some extent by questionnaires, they are considered less objective and trustable (Muir et al., 2016). The study attempts to measure the learner's process emotions through EEG expression analysis to expand the understanding of the learner's emotions in multimedia learning. The relationships between negative emotions, positive emotions, and brain waves were found by recording the brain waves of the learners throughout the entire learning process. This study has shown that brain wave characteristics in positive emotions are significantly higher than those in negative emotions. This is consistent with previous research studies in psychology. Klimesch has demonstrated that the alpha band can reflect the brain's attention processing when performing tasks (Huang et al., 2010). People tend to be more relaxed under positive emotions, so their alpha band will have a greater response. The research done by Cole and Ray (1985) has also shown that the beta and gamma bands have higher energy responses when the brain processes positive emotions.

\section{New Teaching Methods Are Created to Broaden the Scope of Teaching Virtual Reality Immersive Geography Learning}

Several recommendations concerning the implementation of VR immersive geography learning as an active pedagogy can be made using the findings of this pilot study. The exploratory pilot study addresses the gap in the literature examining the application of immersive VR geography learning as an active pedagogy in geography education. One of the biggest challenges facing instructional designers of virtual reality learning environments is how to integrate virtual reality features into lessons to motivate students' learning (Cole and Ray, 1985). With the increasing abundance of personalized instructional resources, more knowledge and skills can be placed in immersive virtual reality learning to enhance learners' mind-flow experiences by including more elements in instructional scripts that induce positive emotions, instructional designs that enhance positive emotions and motivation, and focus on multichannel and multimodal cognitive loads.

\section{CONCLUSION}

In this research, we conducted an empirical study about the influence and effect of the novel immersive VR learning, by comparing EEG waveforms and emotion evoked among different learners. The study found that the positive emotional clues produced during VR immersive learning did trigger the change in the learner's mood. The comparison concludes that this instructional approach can significantly enhance learners' motivation, mind-flow experience.

However, this study did not measure the learning effect, and it is impossible to show through quantitative data that this type of teaching method can improve the learning effect of learners. We also need to design an assessment paper or scale specifically to evaluate the teaching effectiveness of this type of teaching method in future research, to conduct a more scientific learning assessment. Virtual reality technology can be applied to the broad scope of earth science research, such as architecture, geographic information, town planning, digitalization, and natural science. VR's application in earth sciences helps people better understand nature and improve their quality of life. Building realistic virtual models can give people feedback and allow them to make timely improvements and fixes to nature, achieving a win-win situation for both people and earth sciences. However, most of the existing apps are not designed and optimized for learning and teaching purposes. They are limited to passive viewing experiences, or 
lack well-developed story structures (Shih and Yang, 2008). The design of emotional cues based on VR immersive learning is still an open research topic. Specifically, some research questions still need to be properly investigated and addressed in the future, such as choosing the frequency and timing of emotional clues transmission, improving learners' learning motivation and flow experience through emotional design, etc.

\section{DATA AVAILABILITY STATEMENT}

The raw data supporting the conclusions of this article will be made available by the authors, without undue reservation.

\section{REFERENCES}

Alfalah, S. F. M., Falah, J. F. M., Alfalah, T., Elfalah, M., Muhaidat, N., Falah, O., et al. (2019). A comparative study between a virtual reality heart anatomy system and traditional medical teaching modalities. Virtual Real. 23, 229-234.

Bucher, J. (2017). Storytelling for Virtual Reality: Methods and Principles for Crafting Immersive Narratives[M]. Milton Park: Routledge.

Burdea, G., and Coiffet, P. (2003). Virtual Reality Technology. Wiley: IEEE Press.

Chandler, C. L., and Connell, J. P. (1987). Children's intrinsic, extrinsic and internalized motivation: a developmental study of children's reasons for liked and disliked behaviours. Br. J. Dev. Psychol. 5, 357-365. doi: 10.1111/j.2044835x.1987.tb01072.x

Chen, J. (2007). Flow in games (and everything else). Commun. ACM 50, 31-34. doi: $10.1037 / \mathrm{adb} 0000435$

Cobb, S., and Fraser, D. S. (2005). "Multimedia Learning in Virtual Reality," in The Cambridge Handbook of Multimedia Learning, ed. R. E. Mayer (Cambridge: Cambridge University Press).

Cole, H. W., and Ray, W. J. (1985). EEG correlates of emotional tasks related to attentional demands. Int. J. Psychophysiol. 3, 33-41. doi: 10.1016/0167-8760(85) 90017-0

Csikszentmihalyi, M. (2014). Toward a Psychology of Optimal Experience[M]//Flow and the Foundations of Positive Psychology. Dordrecht: Springer, 209-226.

Dooley, K. (2017). Storytelling with virtual reality in 360-degrees: a new screen grammar. Stud. Australas. Cine. 11, 161-171. doi: 10.1080/17503175.2017. 1387357

Eisenberg, N., Emde, R., and Hartup, W. W. (1992). Achievement and Motivation: A Social-Developmental Perspective. Cambridge: Cambridge University Press.

Ekman, P. (1992). Facial expressions of emotion: an old controversy and new findings. Philos. Trans. R. Soc. Lond. Ser. B 335, 63-69. doi: 10.1098/rstb.1992. 0008

Ghergulescu, I., and Muntean, C. H. (2014). A novel sensor-based methodology for learner's motivation analysis in game-based learning. Interact. Comput. 26, 305-320. doi: 10.1093/iwc/iwu013

Hardie, P., Darley, A., Carroll, L., Redmond, C., Campbell, A., Jarvis, S., et al. (2020). Nursing \& Midwifery students' experience of immersive virtual reality storytelling: an evaluative study. BMC Nurs. 19:78. doi: 10.1186/s12912-02000471-5

Herweg, N. A., Solomon, E. A., and Kahana, M. J. (2020). Theta oscillations in human memory. Trends Cogn. Sci. 24, 208-227.

Huang, H. M., Rauch, U., and Liaw, S. S. (2010). Investigating learners' attitudes toward virtual reality learning environments: based on a constructivist approach. Comput. Educ. 55, 1171-1182. doi: 10.1016/j.compedu.2010. 05.014

Inoue, Y. (1999). Effects of Virtual Reality Support Compared to Video Support in a High-School World Geography Class. Campus Wide Inform. Sys. 16, 95-104. doi: $10.1108 / 10650749910281250$

Kleih, S. C., Nijboer, F., Halder, S., and Kübler, A. (2010). Motivation modulates the P300 amplitude during brain-computer interface use. Clin. Neurophysiol. 121, 1023-1031. doi: 10.1016/j.clinph.2010.01.034

\section{ETHICS STATEMENT}

The studies involving human participants were reviewed and approved by the Beijing Normal University. The patients/participants provided their written informed consent to participate in this study.

\section{AUTHOR CONTRIBUTIONS}

YS: write the thesis, organize, and coordinate. ZW: write the thesis and literature research. ML and JY: data analysis. YG: data analysis and literature research. All authors contributed to the article and approved the submitted version.

Knörzer, L., Brünken, R., and Park, B. (2016). Facilitators or suppressors: effects of experimentally induced emotions on multimedia learning. Learn. Instruct. 44, 97-107. doi: 10.1016/j.learninstruc.2016.04.002

Kumar, J. A., Muniandy, B., and Yahaya, W. A. J. W. (2016). Emotional design in multimedia learning: how emotional intelligence moderates learning outcomes. Int. J. Modern Educ. Comput. Sci. 8:54. doi: 10.5815/ijmecs.2016. 05.07

Li, B. J., Bailenson, J. N., Pines, A., and Greenleaf, W. J. (2017). A public database of immersive VR videos with corresponding ratings of arousal, valence, and correlations between head movements and self-report measures. Front. Psychol. 8:2116. doi: 10.3389/fpsyg.2017.02116

Li, W., Gong, J., Wang, D., Huang, M., and Zhou, J. (2007). “Distance Geographic Learning Based on Collaborative Virtual Environment, in Conference: Computational Science - ICCS 2007, 7th International Conference, Beijing, China, May 27 - 30, 2007, Proceedings, Part III. (Berlin: Springer), 516-522. doi: $10.1089 /$ tmj.2009.0057

Liew, T. W., and Tan, S. M. (2016). The effects of positive and negative mood on cognition and motivation in multimedia learning environment. J. Edu. Technol. Soc. 19, 104-115

Limniou, M., Roberts, D., and Papadopoulos, N. (2008). Full immersive virtual environment CAVETM in chemistry education. Comput. Educ. 51, 584-593. doi: 10.1016/j.compedu.2007.06.014

Mayer, R. E. (2010). Applying the science of learning to medical education. Med. Educ. 44, 543-549. doi: 10.1111/j.1365-2923.2010.03624.x

Mayer, R. E. (2013). Emotional design in multimedia learning. Inf. Des. J. Doc. Des. 20, 179-179.

Mayer, R. E. (2014). Incorporating motivation into multimedia learning. Learn. Instruct. 29, 171-173. doi: 10.1016/j.learninstruc.2013.0 4.003

Moreau, K. A., Eady, K., Sikora, L., and Horsley, T. (2018). Digital storytelling in health professions education: a systematic review. BMC Med. Educ. 18:208. doi: 10.1186/s12909-018-1320-1

Muir, K., Madill, A., and Brown, C. (2016). Individual differences in emotional processing and autobiographical memory: Interoceptive awareness and alexithymia in the fading affect bias. Cogn. Emot. 31, 1-13.

Münchow, H., Mengelkamp, C., and Bannert, M. (2017). The better you feel the better you learn: do warm colours and rounded shapes enhance learning outcomes in multimedia learning? Educ. Res. Int. 2017:1.

Pallavicini, F., and Pepe, A. (2020). Virtual reality games and the role of body involvement in enhancing positive emotions and decreasing anxiety: within-subjects pilot study. JMIR Ser. Games 8:e15635. doi: 10.2196/1 5635

Park, B., Knörzer, L., Plass, J. L., and Brünken, R. (2015). Emotional design and positive emotions in multimedia learning: an eyetracking study on the use of anthropomorphisms. Comput. Educ. 86, 30-42. doi: 10.1016/j.compedu.2015. 02.016

Pavelescu, L. M. (2019). Motivation and emotion in the EFL learning experience of Romanian adolescent students: two contrasting cases. Stud. Sec. Lang. Learn. Teach. 9, 55-82. doi: 10.14746/ssllt.2019.9.1.4 
Pi, Z., Zhang, Y., Zhou, W., Xu, K., Chen, Y., Yang, J., et al. (2021). Learning by explaining to oneself and a peer enhances learners' theta and alpha oscillations while watching video lectures. Br. J. Educ. Technol. 52, 659-679. doi: 10.1111/ bjet. 13048

Pintrich, P. R. (1991). A manual for the use of the Motivated Strategies for Learning Questionnaire (MSLQ). Ann Arbor, MI: University of Michigan.

Plass, J. L., Heidig, S., Hayward, E. O., Homer, B. D., and Um, E. (2014). Emotional design in multimedia learning: effects of shape and color on affect and learning. Learn. Instruct. 29, 128-140. doi: 10.1016/j.learninstruc.2013.0 2.006

Rauch, U. (2007). Who owns this space anyway? The Arts 3D VL Metaverse as a network of imagination[C]//EdMedia+ Innovate Learning. Assoc. Adv. Comput. Educ. 2007, 4249-4253.

Roseman, I. J. (1996). Appraisal determinants of emotions: constructing a more accurate and comprehensive theory. Cogn. Emot. 1, 241-278. doi: 10.1111/opn. 12227

Shen, Y., Wang, Z., Hao, A., Peng, Y., Zhai, X., Wang, H., et al. (2021). Investigating the Effect of VR+ Haptics Approach on Students. Complexity 2021, 08-31. doi: 10.1002/jdd.12037

Shih, Y. C., and Yang, M. T. (2008). A collaborative virtual environment for situated language learning using VEC3D. J. Educ. Technol. Soc. 11, 56-68.

Storr, W. (2020). The Science of Storytelling: Why Stories Make Us Human and How to Tell Them Better. New York, NY: Abrams.

Suh, A., and Prophet, J. (2018). The state of immersive technology research: a literature analysis. Comput. Hum. Behav. 86, 77-90.

Thomas, A., Kumar, A., Krehel, R., Vasey, K., Khoo, E., Marsh, T., et al. (2018). Oceans we make: immersive VR storytelling[M]//SIGGRAPH Asia 2018. Virtual Augment. Real. 13, 1-2.

Vansteenkiste, M., Niemiec, C. P., and Soenens, B. (2012). Book Series: Advances in Motivation and Achievement. Bingley: Emerald Group Publishing Limited.

Virvou, M., and Katsionis, G. (2008). On the usability and likeability of virtual reality games for education: the case of VR-ENGAGE[J]. Comput. Educ. 50, $154-178$.
Wang, L. C., and Chen, M. P. (2010). The effects of game strategy and preferencematching on flow experience and programming performance in game-based learning. Innov. Educ. Teach. Int. 47, 39-52.

Weiner, B. (1986). An Attributional Theory of Achievement and Emotion. New York, NY: SpringerVerlag.

Zhai, X., Asmi, F., Yuan, J., Anwar, M. A., Siddiquei, N. L., Ahmad, I., et al. (2021a). The Role of Motivation and Desire in Explaining Students' VR Games Addiction: a Cognitive-Behavioral Perspective. Math. Probl. Eng. 2021:5526046.

Zhai, X., Chu, X., Chai, C. S., Jong, M. S. Y., Istenic, A., Yuan, J., et al. (2021b). A Review of Artificial Intelligence (AI) in Education from 2010 to 2020. Complexity 2021:8812542.

Zheng, W. L., and Lu, B. L. (2015). Investigating critical frequency bands and channels for EEG-based emotion recognition with deep neural networks. IEEE Trans. Auton. Men. Dev. 7, 162-175.

Conflict of Interest: The authors declare that the research was conducted in the absence of any commercial or financial relationships that could be construed as a potential conflict of interest.

Publisher's Note: All claims expressed in this article are solely those of the authors and do not necessarily represent those of their affiliated organizations, or those of the publisher, the editors and the reviewers. Any product that may be evaluated in this article, or claim that may be made by its manufacturer, is not guaranteed or endorsed by the publisher.

Copyright (c) 2022 Shen, Wang, Li, Yuan and Gu. This is an open-access article distributed under the terms of the Creative Commons Attribution License (CC BY). The use, distribution or reproduction in other forums is permitted, provided the original author(s) and the copyright owner(s) are credited and that the original publication in this journal is cited, in accordance with accepted academic practice. No use, distribution or reproduction is permitted which does not comply with these terms. 